Article

\title{
Graphene-Based Cylindrical Pillar Gratings for Polarization-Insensitive Optical Absorbers
}

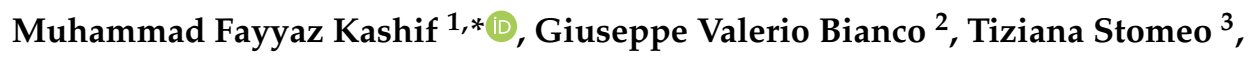 \\ Maria Antonietta Vincenti ${ }^{4}{ }^{(0)}$, Domenico de Ceglia ${ }^{5}$, Massimo De Vittorio ${ }^{3,6}$, Michael Scalora ${ }^{7}$, \\ Giovanni Bruno ${ }^{2}$, Antonella D'Orazio ${ }^{1}$ and Marco Grande ${ }^{1,2}$ \\ 1 Dipartimento di Ingegneria Elettrica e dell'Informazione, Politecnico di Bari, Via Orabona 4, 70125 Bari, Italy; \\ antonella.dorazio@poliba.it (A.D.); marco.grande@poliba.it (M.G.) \\ 2 Istituto di Nanotecnologia-CNR-NANOTEC, Via Orabona 4, 70125 Bari, Italy; \\ giuseppevalerio.bianco@cnr.it (G.V.B.); giovanni.bruno@cnr.it (G.B.) \\ 3 Center for Bio-Molecular Nanotechnology, Istituto Italiano di Tecnologia (IIT), Via Barsanti, 73010 Arnesano, \\ Lecce, Italy; tiziana.stomeo@iit.it (T.S.); massimo.devittorio@iit.it (M.D.V.) \\ 4 Department of Information Engineering, University of Brescia, Via Branze 38, 25121 Brescia, Italy; \\ maria.vincenti@unibs.it \\ 5 Department of Information Engineering, University of Padova, Via Gradenigo 6/b, 35131 Padova, Italy; \\ domenico.deceglia@unipd.it \\ 6 Dipartimento di Ingegneria dell'Innovazione, Università del Salento, Via Arnesano, 73100 Lecce, Italy \\ 7 Charles M. Bowden Research Center, RDECOM, Redstone Arsenal, Huntsville, AL 35898-5000, USA; \\ michael.scalora.civ@mail.mil \\ * Correspondence: muhammadfayyaz.kashif@poliba.it
}

Received: 23 May 2019; Accepted: 18 June 2019; Published: 21 June 2019

\begin{abstract}
In this study, we present a two-dimensional dielectric grating which allows achieving high absorption in a monolayer graphene at visible and near-infrared frequencies. Dielectric gratings create guided-mode resonances that are exploited to effectively couple light with the graphene layer. The proposed structure was numerically analyzed through a rigorous coupled-wave analysis method. Effects of geometrical parameters and response to the oblique incidence of the plane wave were studied. Numerical results reveal that light absorption in the proposed structure is almost insensitive to the angle of the impinging source over a considerable wide angular range of $20^{\circ}$. This may lead to the development of easy to fabricate and experimentally viable graphene-based absorbers in the future.
\end{abstract}

Keywords: diffraction grating; guided mode resonance; graphene; absorption

\section{Introduction}

Graphene has emerged as an attractive two-dimensional material due to its exceptional mechanical, electrical, and optical properties [1]. Monolayer graphene exhibits better absorbance than different dielectric and metals of the same thickness. A considerable amount of theoretical and experimental work has been published in recent years to explore the potential of graphene in optical and microwave absorbers in different spectral ranges of the electromagnetic spectrum for various applications such as biosensors, optical filters, modulators, and efficient photodetector [2-4].

Monolayer graphene shows a constant absorption of about 2.3\% over the visible and near-infrared wavelengths [5]. However, in the context of optical absorbers, this value is not enough which substantially limits its use in photonic devices. Therefore, enhancement of light absorption by monolayer graphene has drawn significant attention from researchers and several solutions have been proposed in the technical literature to achieve the perfect absorption. One- and two-dimensional 
dielectric gratings exploit guided-mode resonance (GMR) to increase light-matter interaction. Light is effectively coupled to graphene if incorporated with such gratings [6]. By varying geometrical parameters of the grating structure, the resonant wavelength and the bandwidth of absorption are tuned. A number of numerical studies [7-10] report perfect absorption of light in graphene incorporated dielectric gratings over diverse spectral ranges. Recently, near total absorption in monolayer graphene based on critical coupling was presented at a visible wavelength of $605 \mathrm{~nm}$ [11]. In some other recent studies, multilevel structures were investigated and enhanced graphene absorption was reported [12-14]. The main drawback of the multilevel structures is the complexity of the fabrication process since it requires the deposition of multiple material layers. Experimental and measured results for several fabricated graphene-based structures have also been presented [15-18]. The maximum experimentally measured values of absorption reported are $35 \%$ at $0.73 \mu \mathrm{m}$ [15], $40 \%$ at $0.7 \mu \mathrm{m}$ [16], $45 \%$ at $10 \mu \mathrm{m}$ [17], and $99 \%$ at $1.48 \mu \mathrm{m}$ [18]. Apart from grating-based structures, perfect absorbers based on metamaterials have also been reported [19-22].

However, perfect, broadband, tunable and polarization-independent absorption of light in monolayer graphene for photonic applications is still a challenging problem and needs further research and exploration. In this framework, it is worth pointing out that the absorption spectra of resonant gratings are highly sensitive to the angle of incident light.

In previous work, we demonstrated that graphene-based gratings are exploited to modulate the Fano-like signature of GMRs [23]. Moreover, two-dimensional arrays of rectangular gold nanopatches grown on monolayer graphene were used to experimentally demonstrate the polarization dependence of plasmonic gratings at normal incidence [24].

In this study, we numerically investigate a two-dimensional (2D) dielectric grating through the rigorous coupled-wave analysis (RCWA) method. We demonstrate the effects of geometrical parameters of the 2D grating on its spectral characteristics of interest. We further study the response of the proposed device for the oblique incidence of a plane wave source. We show that it has a stable optical absorption of around $40 \%$ over a considerable wide angular range of $20^{\circ}$. Electromagnetic field distributions at resonant frequencies are presented to explain the underlying physical phenomenon for optical absorption.

\section{Materials and Methods}

Figure 1a shows the sketch of the proposed 2D dielectric grating. It consisted of a periodic array of polymethyl-methacrylate (PMMA) cylindrical pillars deposited on a tantalum pentoxide $\left(\mathrm{Ta}_{2} \mathrm{O}_{5}\right)$ waveguide. A slab of silicon dioxide $\left(\mathrm{SiO}_{2}\right)$ was used as a substrate. The monolayer graphene was placed on top of the waveguide under the PMMA pillars. The proposed 2D dielectric grating can be realized using nanoimprint lithography (NIL). This technology enables the faster fabrication of polymeric-based dielectric gratings at a low cost. The choice of the proposed 2D configuration was based on the possibility of an insensitive polarization behavior at normal incidence for the GMRs that are excited in the $\mathrm{Ta}_{2} \mathrm{O}_{5}$ dielectric waveguide. A $20 \mathrm{~nm}$ thick buffer layer of PMMA was added on top of the graphene layer (and under the cylinder) to take into account the fabrication tolerances introduced by the NIL fabrication step.

The thickness of the monolayer graphene was equal to $0.34 \mathrm{~nm}$. A thin buffer layer of PMMA (20 nm thick) was also placed over the monolayer graphene considering the quality of graphene was not affected by pillar fabrication. The geometrical parameters of the device are detailed in Figure $1 \mathrm{~b}$. The thickness $t_{T a 2 O 5}$ of the $\mathrm{Ta}_{2} \mathrm{O}_{5}$ slab and the PMMA pillar height $t_{P M M A}$ were initially set equal to $150 \mathrm{~nm}$ and $600 \mathrm{~nm}$, respectively. The PMMA pillars have symmetric periodicity both in $x$ - and $y$ directions and the value chosen was equal to $600 \mathrm{~nm}$ at the start. The radius $r$ of the cylindrical PMMA pillars was chosen as $250 \mathrm{~nm}$. 


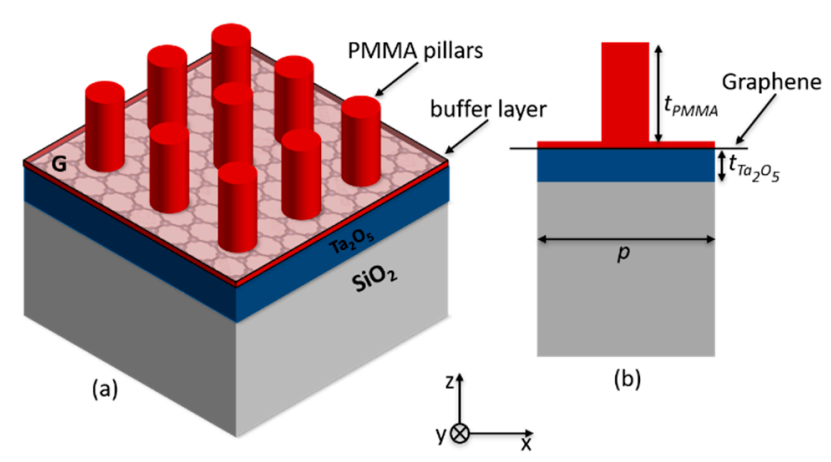

Figure 1. (a) Sketch of a 2D graphene-based dielectric grating with an array of cylindrical pillars and (b) the cross-sectional $(x, z)$ view of the single period of the device used for simulations.

The 2D graphene-based dielectric gratings were simulated by means of RSoft-DiffactMOD that implements a Fourier-space method, the rigorous coupled-wave analysis (RCWA), to solve scattering from periodic structures. The refractive indices for $\mathrm{PMMA} \mathrm{Ta}_{2} \mathrm{O}_{5}$, and $\mathrm{SiO}_{2}$ were retrieved from Palik et al. [25]. For monolayer graphene, we used the complex refractive index $n=3+\mathrm{jC}_{1} * \lambda / 3$ reported in [26] at visible frequencies where $C_{1}$ was equal to $5.446 \mu \mathrm{m}^{-1}$ and $\lambda$ was the free space wavelength to take into account the losses.

\section{Results}

Figure 2 shows the reflection, transmission, and absorption spectra of the device with and without graphene layer for the TE and TM polarizations at normal incidence. The absorption spectrum shows resonant peaks at the $0.7 \mu \mathrm{m}, 0.9 \mu \mathrm{m}$, and $0.96 \mu \mathrm{m}$ wavelengths. These correspond to absorption of $20 \%, 50 \%$, and $40 \%$, respectively. The absorption is zero when the structure has no graphene layer. It is noted that the optical response is insensitive to the polarization of the incoming light wave due to the symmetry of the device (2D square lattice).

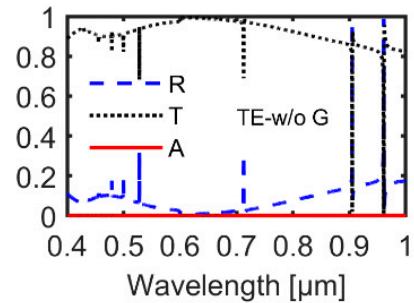

(a)

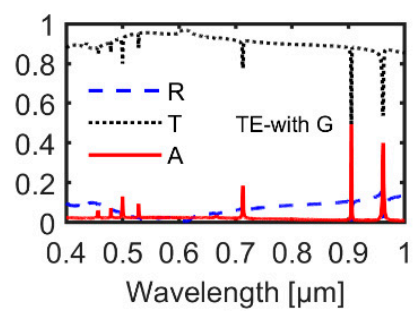

(c)

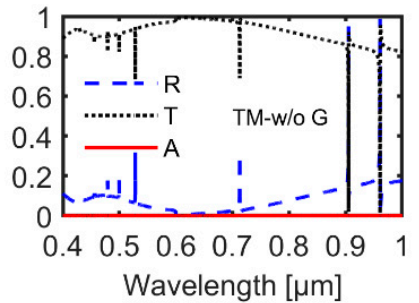

(b)

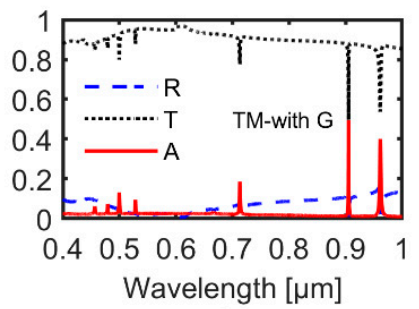

(d)

Figure 2. Optical reflectance (dashed blue line), the transmittance (dotted black line), and absorption (solid red line) spectra $(\mathbf{a}, \mathbf{b})$ without and $(\mathbf{c}, \mathbf{d})$ with graphene layer for both $(\mathbf{a}, \mathbf{c})$ TE and $(\mathbf{b}, \mathbf{d})$ TM plane waves at normal incidence. The device period $\mathrm{p}$, waveguide $\mathrm{Ta}_{2} \mathrm{O}_{5}$ thickness $\mathrm{t}_{\mathrm{Ta2}} \mathrm{O} 5$, and PMMA pillars height $t_{\text {PMMA }}$ for this simulation result are set as $600 \mathrm{~nm}, 150 \mathrm{~nm}$, and $600 \mathrm{~nm}$, respectively.

The electromagnetic field distributions are shown in Figure $3 a, b$ at the resonant wavelength of $0.9 \mu \mathrm{m}$ (maximum absorption) for the TE and TM polarizations, respectively. It clearly illustrates that the fields are concentrated around monolayer graphene. The white lines represent the device structure 
where the monolayer graphene is positioned at $z=0.15 \mu \mathrm{m}$ (that corresponds to the thickness of the $\mathrm{Ta}_{2} \mathrm{O}_{5}$ ). The comparison between Figure 3 (with graphene) and Figure 4 (without graphene) shows the difference between the values of field magnitudes of about a factor two.
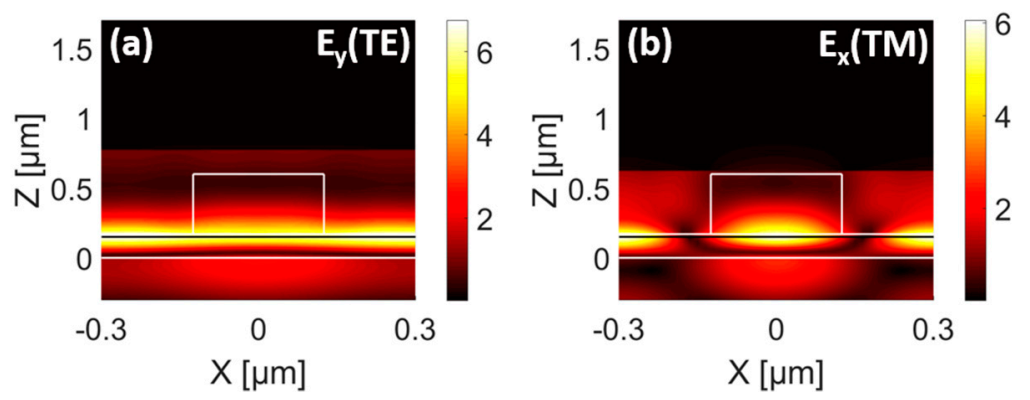

Figure 3. Field profiles (a) amplitude of Ey component and (b) amplitude of Ex component at $0.9 \mu \mathrm{m}$ for the device parameters of Figure 2. The white lines show the device structure where the monolayer graphene is positioned at $z=0.15 \mu \mathrm{m}$ (marked by the black line).
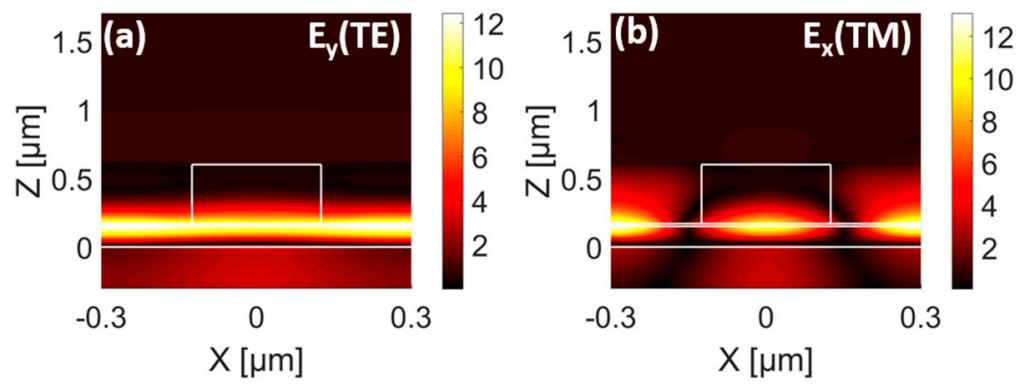

Figure 4. Field profiles (a) amplitude of Ey component and (b) amplitude of Ex component at $0.9 \mu \mathrm{m}$ without the graphene layer. The white lines show the device structure.

Next, we studied the effects of device geometrical parameters. Figure 5 describes the absorption (A) spectra at different grating periods $\left(\mathrm{p}\right.$ ) while $\mathrm{Ta}_{2} \mathrm{O}_{5}$ layer thickness $t_{T a 2} \mathrm{O}_{5}$ and PMMA pillar height $t_{P M M A}$ are kept constant. It is evident that absorption peak wavelength is tuned over the visible and near-infrared wavelengths by changing the grating period. The sliding of the resonant wavelengths with increasing device period agrees well with the analysis presented in [6]. Each absorption spectrum shows multiple peaks which result from multiple guided-mode resonances. In addition, there is an increase in absorption with increasing period length as is observed in Figure 5a-d. Since our device is polarization independent for normally incident plane waves, we have shown the absorption spectra for TE polarized plane waves only.

The effects of variations of $\mathrm{Ta}_{2} \mathrm{O}_{5}$ layer thickness $t_{T a 2 O 5}$ and PMMA pillar height $t_{P M M A}$ on the absorption spectrum have also been investigated. Figure 6a depicts the effect of change of $t_{T a 2 O 5}$ on the absorption spectrum. There is a red shift in the resonant wavelength as the thickness of the $\mathrm{Ta}_{2} \mathrm{O}_{5}$ waveguide is increased from $80 \mathrm{~nm}$ to $160 \mathrm{~nm}$. Multiple resonances appear among which the prominent ones are shown in the absorption map.

Figure $6 \mathrm{~b}$ depicts the effect of change of $t_{P M M A}$ on the absorption spectrum revealing no changes when the PMMA thickness is varied in the range of $300 \mathrm{~nm}$ to $900 \mathrm{~nm}$. 


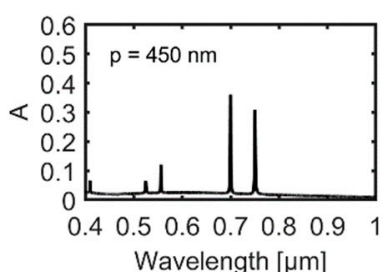

(a)

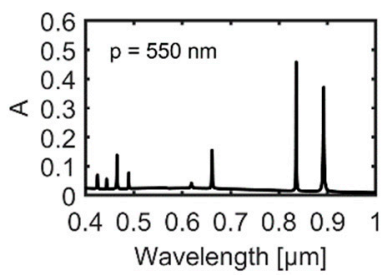

(c)

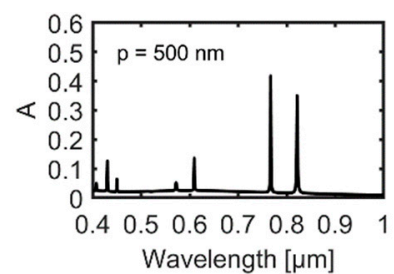

(b)

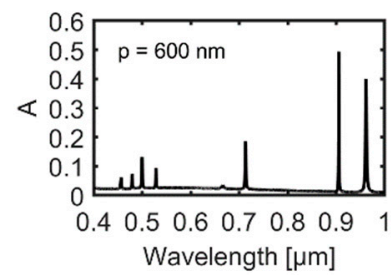

(d)

Figure 5. Absorption spectra of the proposed structure under varying grating period for normally incident TE polarized plane wave. The waveguide $\mathrm{Ta}_{2} \mathrm{O}_{5}$ thickness $\mathrm{t}_{\mathrm{Ta} 2 \mathrm{O} 5}$, and polymethyl-methacrylate (PMMA) pillars height $t_{\text {PMMA }}$ are fixed at $150 \mathrm{~nm}$ and $600 \mathrm{~nm}$, respectively.
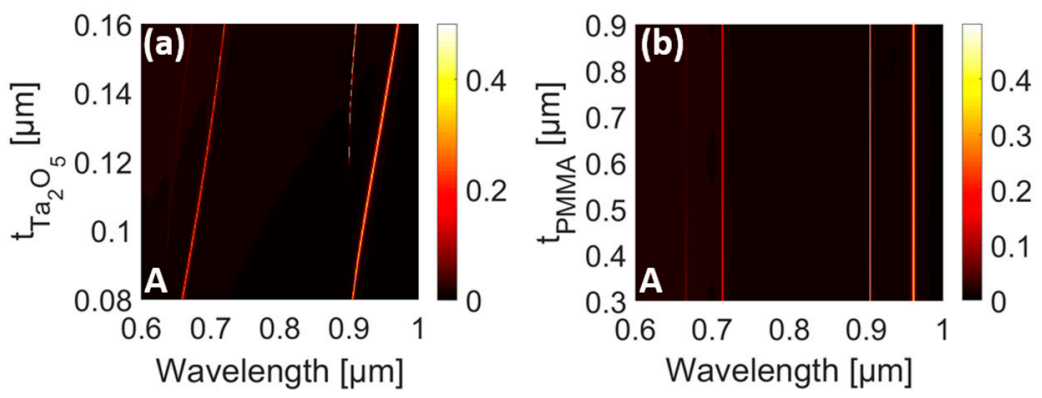

Figure 6. (a) Absorption map with respect to the variation of $\mathrm{Ta}_{2} \mathrm{O}_{5}$ layer thickness $\mathrm{t}_{\mathrm{Ta} 2 \mathrm{O} 5}$ under $\mathrm{TE}$ at normal illumination when the period, $\mathrm{p}$, and PMMA pillar height, $\mathrm{t}_{\mathrm{PMMA}}$, are fixed at $600 \mathrm{~nm}$ and 600 nm, respectively. (b) Absorption map under TE normal illumination, when PMMA pillar thickness $t_{\text {PMMA }}$ is varied from $300 \mathrm{~nm}$ to $900 \mathrm{~nm}$. The period, $\mathrm{p}$, and waveguide, $\mathrm{Ta}_{2} \mathrm{O}_{5}$, thickness $\mathrm{t}_{\mathrm{Ta} 2 \mathrm{O} 5}$ are fixed at $600 \mathrm{~nm}$ and $150 \mathrm{~nm}$, respectively.

We further analyze the optical response of the proposed device under the oblique incidence of the plane wave source. Figure 7 shows the absorption maps when the incidence angle is changed from $0^{\circ}$ to $90^{\circ}$ for both TE and TM polarizations. The maps show that the resonance at normal incidence splits into two arms for angular incidence. This behavior is typical of a grating structure that is derived by the absorption spectra. It is interesting to note that the absorption spectra do not split into two arms for certain resonant wavelengths and absorption is nearly independent of the source angle for specific angular ranges. 

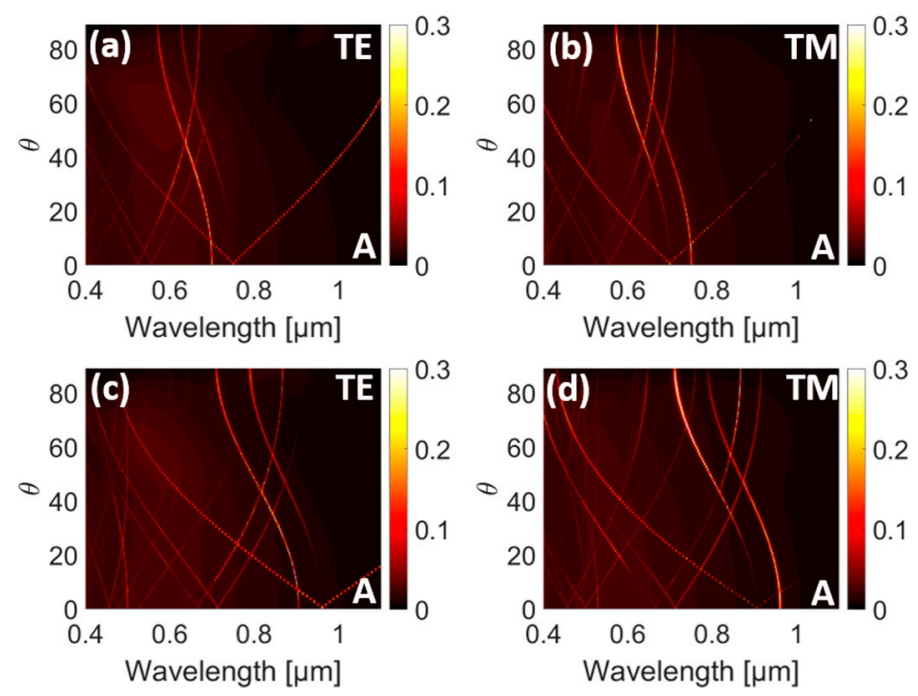

Figure 7. Absorption maps of the device for varying incidence angle (0-90 $)$ under both TE and TM polarizations. For $(\mathbf{a}, \mathbf{b})$ device period $\mathrm{p}$, the waveguide $\mathrm{Ta}_{2} \mathrm{O}_{5}$ thickness $\mathrm{t}_{\mathrm{Ta} 2 \mathrm{O} 5}$, and PMMA pillars height $t_{\text {PMMA }}$ are fixed at $450 \mathrm{~nm}, 150 \mathrm{~nm}$, and $600 \mathrm{~nm}$, respectively, whereas, for $(\mathbf{c}, \mathbf{d})$ the period $\mathrm{p}$ is changed to $600 \mathrm{~nm}$.

To further elaborate these important results, the absorption spectra of the device for both TE and TM polarizations are shown in Figure 8 in an angular range of $0-20^{\circ}$ for two different device periods. The absorption is limited within narrow bandwidths of $\sim 15 \mathrm{~nm}$ and $\sim 20 \mathrm{~nm}$ for the maps in $(\mathrm{a}, \mathrm{b})$ and $(\mathrm{c}, \mathrm{d})$ respectively over a considerable wide angular range of $20^{\circ}$. The slope is about $0.5 \mathrm{~nm} /$ degree in the $0-10^{\circ}$ range.

Another important result is depicted in Figure 9 which shows absorption spectra in an angular range of $70-90^{\circ}$. It is noted that the absorption is limited in narrow bandwidths of $\sim 25 \mathrm{~nm}$ and $\sim 10 \mathrm{~nm}$ for the angular ranges of $70-90^{\circ}$ and $75-90^{\circ}$, respectively in both (a) and (b) maps. Furthermore, the device behavior is similar under both TE and TM polarizations.
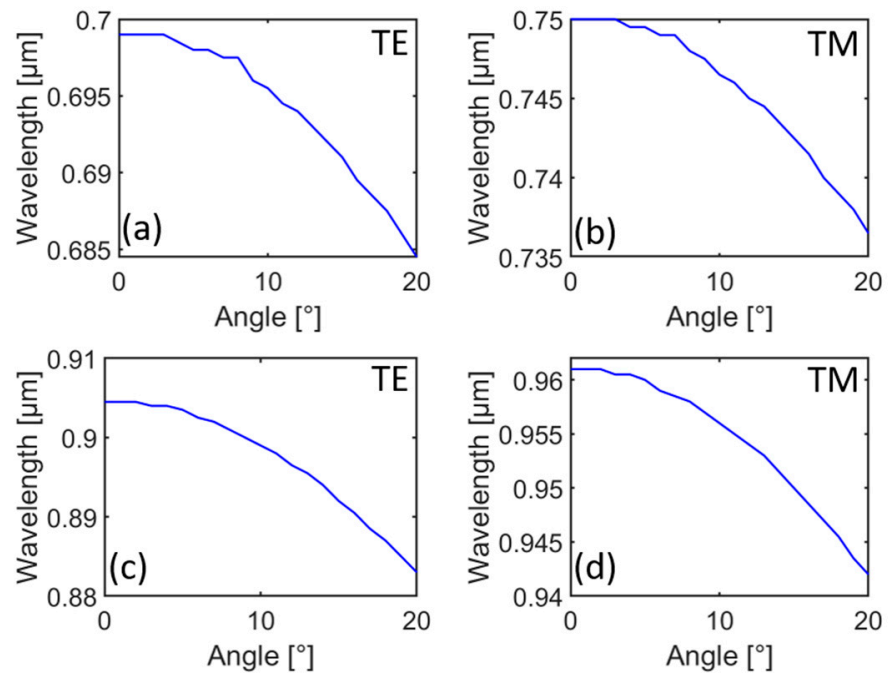

Figure 8. Peak absorption wavelength when the incident angle is varied in range $0-20^{\circ}$. $(\mathbf{a}, \mathbf{b})$ The device period $\mathrm{p}$, the waveguide $\mathrm{Ta}_{2} \mathrm{O}_{5}$ thickness $\mathrm{t}_{\mathrm{Ta} 2 \mathrm{O}}$, and PMMA pillars height $\mathrm{t}_{\text {PMMA }}$ are fixed at $450 \mathrm{~nm}, 150 \mathrm{~nm}$, and $600 \mathrm{~nm}$, respectively. (c,d) The device period p, the waveguide $\mathrm{Ta}_{2} \mathrm{O}_{5}$ thickness $\mathrm{t}_{\mathrm{Ta} 2 \mathrm{O} 5}$, and PMMA pillars height $\mathrm{t}_{\mathrm{PMMA}}$ are fixed at $600 \mathrm{~nm}, 150 \mathrm{~nm}$, and $600 \mathrm{~nm}$, respectively. 
In short, the observations in Figure 8; Figure 9 prove the near insensitivity of the proposed device to the angle and polarization of the incident light in multiple angular ranges of around $20^{\circ}$. Angular response of our device shows clear improvement as compared with results reported in [15] for a graphene-based absorber based on a one-dimensional grating where absorption spectra split into two arms from $0-20^{\circ}$ under similar simulation conditions.
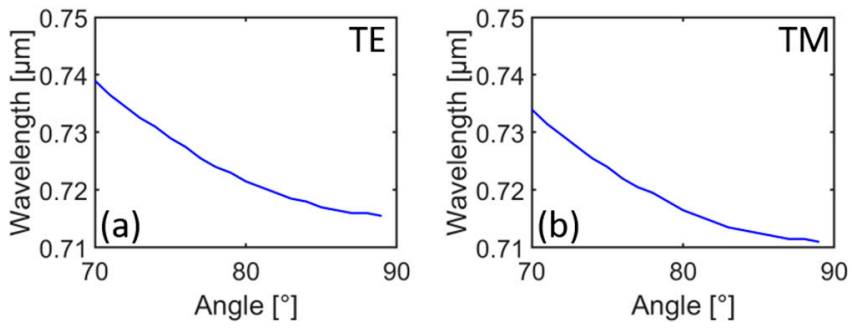

Figure 9. Peak absorption wavelength of the device for varying incidence angle (70-90 $)$ under both TE and TM polarizations. The device period $\mathrm{p}$, the waveguide $\mathrm{Ta}_{2} \mathrm{O}_{5}$ thickness $\mathrm{t}_{\mathrm{Ta} 2 \mathrm{O} 5}$, and PMMA pillars height $\mathrm{t}_{\mathrm{PMMA}}$ are fixed at $600 \mathrm{~nm}, 150 \mathrm{~nm}$, and $600 \mathrm{~nm}$, respectively.

\section{Discussion}

In our previous work [15,16], we experimentally demonstrated light absorption in graphene-based one-dimensional dielectric gratings based on PMMA rectangular strips. However, the response of the structures is sensitive to the oblique incidence of light. In this study, we propose a device based on a 2D grating of cylindrical PMMA pillars that exploit GMRs to enhance light absorption in the monolayer graphene. Our proposed structure achieved light absorption of more than $40 \%$ at multiple wavelengths for both TE and TM polarizations over narrow bandwidths in the visible and near-infrared ranges. The device performance is insensitive to variation in the PMMA pillar height which is an important measure in terms of device stability and fabrication tolerances.

Our simulations further revealed that the device has a considerable stable angular response. The absorption spectra are nearly independent of the angle of the impinging light up to $20^{\circ}$ with a slope of $\sim 0.5 \mathrm{~nm} /$ degree. Moreover, the response is less sensitive to the polarization of the incident plane waves.

In conclusion, the proposed optical absorber is less sensitive to geometrical parameters variations, polarization, and angle of the incident light which can be easily realized using nanofabrication technologies such as nanoimprint lithography.

These results pave the way to the realization of polarization-insensitive optical absorbers that can be efficiently exploited in several applications and photonic devices such as biosensors, optical filters, modulators, and efficient photodetectors.

Author Contributions: M.F.K. prepared the original draft. G.V.B., T.S., M.A.V., D.d.C., M.D.V., M.S., G.B., A.D., and M.G. contributed to the review and editing of the paper. M.G. supervised the research activity.

Funding: Research was sponsored by the RDECOM, RFEC-Atlantic, and the US Army Aviation and Missile Research, Development and Engineering Center and was accomplished under Grant Numbers W911NF-16-2-0236. The views and conclusions contained in this document are those of the authors and should not be interpreted as representing the official policies, either expressed or implied, of the AMRDEC or the U.S. Government.

Conflicts of Interest: The authors declare no competing interests.

\section{References}

1. Novoselov, K.S.; Geim, A.K.; Morozov, S.V.; Jiang, D.; Zhang, Y.; Dubonos, S.V.; Grigorieva, I.V.; Firsov, A.A. Electric field effect in atomically thin carbon films. Science 2004, 306, 666-669. [CrossRef] [PubMed]

2. Szunerits, S.; Boukherroub, R. Graphene-based biosensors. Interface Focus 2018, 8, 20160132. [CrossRef] [PubMed]

3. Freitag, M.; Low, T.; Zhu, W.; Yan, H.; Xia, F.; Avouris, P. Photocurrent in graphene harnessed by tunable intrinsic plasmons. Nat. Commun. 2013, 4, 1951-1958. [CrossRef] [PubMed] 
4. Zhenhua, S.; Haixin, C. Graphene and graphene-like two-dimensional materials in photodetection: Mechanisms and methodology. ACS Nano 2014, 8, 4133-4156. [CrossRef]

5. Butler, S.Z.; Hollen, S.M.; Cao, L.; Cui, Y.; Gupta, J.A.; Gutiérrez, H.R.; Heinz, T.F.; Hong, S.S.; Huang, J.; Ismach, A.F.; et al. Progress, challenges, and opportunities in two-dimensional materials beyond graphene. ACS Nano 2013, 7, 2898-2926. [CrossRef]

6. Gao, W.; Shu, J.; Qiu, C.; Xu, Q. Excitation of plasmonic waves in graphene by guided-mode resonances. ACS Nano 2012, 6, 7806-7813. [CrossRef]

7. Fan, Y.; Guo, C.; Zhu, Z.; Xu, W.; Wu, F.; Yuan, X.; Qin, S. Monolayer-graphene-based broadband and wide-angle perfect absorption structures in the near infrared. Sci. Rep. 2018, 8, 13709. [CrossRef]

8. Zhang, S.; Wang, Y.; Wang, S.; Zheng, W. Wavelength-tunable perfect absorber based on guided-mode resonances. Appl. Opt. 2016, 55, 3176-3181. [CrossRef]

9. Zhao, B.; Zhao, J.M.; Zhang, Z.M. Resonance enhanced absorption in a graphene monolayer using deep metal gratings. J. Opt. Soc. Am. B 2015, 32, 1176-1185. [CrossRef]

10. Vincenti, M.A.; de Ceglia, D.; Grande, M.; D'Orazio, A.; Scalora, M. Nonlinear control of absorption in one-dimensional photonic crystal with graphene-based defect. Opt. Lett. 2013, 38, 3550-3553. [CrossRef]

11. Mahigir, A.; Veronis, G. Nanostructure for near total light absorption in a monolayer of graphene in the visible. J. Opt. Soc. Am. B 2018, 35, 3153-3158. [CrossRef]

12. Wu, J. Ultra-narrow perfect graphene absorber based on critical coupling. Opt. Commun. 2019, 435, 25-29. [CrossRef]

13. Yang, L.; Wang, J.; Lu, C. Sensitive perfect absorber with monolayer graphene-based multi-layer dielectric grating structure. Optik (Stuttg.) 2018, 158, 508-513. [CrossRef]

14. Hu, J.; Qing, Y.; Yang, S.; Ren, Y.; Wu, X.; Gao, W.; Wu, C. Tailoring total absorption in a graphene monolayer covered subwavelength multilayer dielectric grating structure at near-infrared frequencies. J. Opt. Soc. Am. B 2017, 34, 861-868. [CrossRef]

15. Grande, M.; Vincenti, M.A.; Stomeo, T.; Bianco, G.V.; De Ceglia, D.; Aközbek, N.; Petruzzelli, V.; Bruno, G.; De Vittorio, M.; Scalora, M.; et al. Graphene-based perfect optical absorbers harnessing guided mode resonances. Opt. Express 2015, 23, 21032-21042. [CrossRef] [PubMed]

16. Grande, M.; Vincenti, M.A.; Stomeo, T.; Bianco, G.V.; De Ceglia, D.; Aközbek, N.; Petruzzelli, V.; Bruno, G.; De Vittorio, M.; Scalora, M.; et al. Graphene-based absorber exploiting guided mode resonances in one-dimensional gratings. Opt. Express 2014, 22, 31511-31519. [CrossRef] [PubMed]

17. Safaei, A.; Chandra, S.; Vázquez-Guardado, A.; Calderon, J.; Franklin, D.; Tetard, L.; Zhai, L.; Leuenberger, M.N.; Chanda, D. Dynamically tunable extraordinary light absorption in monolayer graphene. Phys. Rev. B 2017, 96, 165431. [CrossRef]

18. Guo, C.C.; Zhu, Z.H.; Yuan, X.D.; Ye, W.M.; Liu, K.; Zhang, J.F.; Xu, W.; Qin, S.Q. Experimental demonstration of total absorption over $99 \%$ in the near infrared for monolayer-graphene-based subwavelength structures. Adv. Opt. Mater. 2016, 4, 1955-1960. [CrossRef]

19. Lin, H.; Sturmberg, B.C.; Lin, K.T.; Yang, Y.; Zheng, X.; Chong, T.K.; de Sterke, C.M.; Jia, B. A 90-nm-thick graphene metamaterial for strong and extremely broadband absorption of unpolarized light. Nat. Photonics 2019, 13, 270-276. [CrossRef]

20. Landy, N.I.; Sajuyigbe, S.; Mock, J.J.; Smith, D.R.; Padilla, W.J. Perfect metamaterial absorber. Phys. Rev. Lett. 2008, 100, 207402. [CrossRef]

21. Xiang, Y.; Dai, X.; Guo, J.; Zhang, H.; Wen, S.; Tang, D. Critical coupling with graphene-based hyperbolic metamaterials. Sci. Rep. 2014, 4, 5483. [CrossRef]

22. Sreekanth, K.V.; ElKabbash, M.; Alapan, Y.; Rashed, A.R.; Gurkan, U.A.; Strangi, G. A multiband perfect absorber based on hyperbolic metamaterials. Sci. Rep. 2016, 6, 26272. [CrossRef]

23. de Ceglia, D.; Vincenti, M.A.; Grande, M.; Bianco, G.V.; Bruno, G.; D’Orazio, A.; Scalora, M. Tuning infrared guided-mode resonances with graphene. J. Opt. Soc. Am. B 2016, 33, 426-433. [CrossRef]

24. Grande, M.; Stomeo, T.; Bianco, G.V.; Vincenti, M.A.; De Ceglia, D.; Petruzzelli, V.; Bruno, G.; De Vittorio, M.; Scalora, M.; D'Orazio, A. Fabrication of doubly resonant plasmonic nanopatch arrays on graphene. Appl. Phys. Lett. 2013, 102, 231111. [CrossRef] 
25. Palik, E.D.; Ghosh, G. Handbook of Optical Constants of Solids, 3rd ed.; Academic Press: San Deigo, CA, USA, 1998.

26. Bruna, M.; Borini, S. Optical constants of graphene layers in the visible range. Appl. Phys. Lett. 2009, 94, 031901. [CrossRef]

(c)

(C) 2019 by the authors. Licensee MDPI, Basel, Switzerland. This article is an open access article distributed under the terms and conditions of the Creative Commons Attribution (CC BY) license (http://creativecommons.org/licenses/by/4.0/). 